\title{
Software project planning through comparison of Bio-inspired algorithms
}

Jesús Silva, Noel Varela, Harold Neira Molina, Omar Bonerge Pineda Lezama

\begin{abstract}
Currently many organizations have adopted the development of software projects with agile methodologies, particularly Scrum, which has more than 20 years of development. In these methodologies, software is developed iteratively and delivered to the client in increments called releases. In the releases, the goal is to develop system functionality that quickly adds value to the client's business. At the beginning of the project, one or more releases are planned. For solving the problem of replanning in the context of releases, a model is proposed considering the characteristics of agile development using Scrum. The results obtained show that the algorithm takes a little less than $7 \mathrm{~min}$ for solutions that propose replanning composed by 16 sprints, which is equivalent to 240 days of project. They show that applying a repair operator increases the hypervolume quality indicator in the resulting population.
\end{abstract}

\section{Palabras clave}

Genetic algorithm, Agile software projects, Multi-target 